\title{
Novel Selective Mapping with Oppositional Hosted Cuckoo Optimization Algorithm for PAPR Reduction in 5G UFMC Systems
}

\author{
A. JAYAMATH ${ }^{*}$, T. JAYASANKAR, K. VINOTH KUMAR
}

\begin{abstract}
In recent times, there is a continuous requirement of achieving high data rates owing to an increase in the number of devices and significant demand for various services with maximum reliability and minimum delay. It results in the development of fifth generation (5G) to offer better services with enhanced data rate. Recently, a major alternative to OFDM technology for $5 \mathrm{G}$ networks called universal filtered multi-carrier (UFMC) is presented where every individual sub-band is filtered that reduces the OOB radiation and eliminates guard band. But high peak-to-average power ratio $(P A P R)$ is a crucial issue which arises from the utilization of several subcarriers to generate the time domain transmission signal. For resolving this issue, this paper presents a novel selective mapping with oppositional hosted cuckoo optimization (SM-OHOCO) algorithm for PAPR reduction in 5G UFMC systems. In the SM-OHOCO algorithm, rather than the generation of several random phase sequences, SM-OHOCO algorithm is performed iteratively to attain a better solution with few searching rounds, showing the novelty of the work. As the optimization of phase sequence in the SLM technique is considered as an NP hard optimization problem, the OHOCO algorithm is applied, which is derived by incorporating the concepts of the HOCO algorithm with oppositional based learning (OBL) strategy. To validate the effective performance of the proposed SM-OHOCO algorithm, an extensive experimental analysis is performed to highlight the improved performance in $5 \mathrm{G}$ networks. The resultant values pointed out the superior outcome of the proposed SM-OHOCO algorithm over the other existing methods in terms of distinct measures
\end{abstract}

Keywords: metaheuristic optimization algorithm; PAPR reduction; selective mapping; universal filtered multi-carrier; $5 \mathrm{G}$ networks

\section{INTRODUCTION}

Fifth generation (5G) mobile communication technique is developed to offer high speed, with improved safety and accessibility than current techniques of transmission between user and machines. [1] The 5G transmission network is significant for wireless connections, which is required to understand the applications like reducing the signal expenses, relaxing synchronization, machine kind transmission, Internet of Things (IoT), and provide better energy effectiveness than current techniques [6]. The presently utilized modulation system, in 4G network, is orthogonal frequency division multiplexing (OFDM). The OFDM utilizes a guard interval and cyclic prefix (CP) among OFDM symbols for avoiding inter symbol interference (ISI) and inter-channel interference (ICI) [5]. Moreover, OFDM has difficult design of power amplifier, larger side lobes, synchronization errors in data aggregation and high peakto-average power ratio $(P A P R)$; consequently, it could not be suggested to use for IoE network and cognitive radios (CR). To resolve this challenge, novel candidate waveforms like universal filtered multi-carrier (UFMC) and filter bank multi-carrier (FBMC) were presented. Fig. 1 shows the general structure of UFMC system. In UFMC method, the subcarriers in every subband preserve an orthogonal connection; additionally, the subbands are filters that could decrease out-of-band (OOB) radiations.

In UFMC method, the subcarriers are filtered and packed as subbands. It decreases the filter length, creating most appropriate UFMC for dynamic spectrum allocation methods in CR networks [21, 22]. Additionally, UFMC offers multi service applications, supports frequency segmentation, low latency, and side lobe radiation. Moreover, it is appropriate for shorter burst transmission. The UFMC utilizes Dolph-Chebyshev filter to all subbands. In the pass-band, the useful signals are accepted with no damage but from the stop-band, the frequency response is decayed faster for avoiding $\mathrm{OOB}$ emission
[24]. It is noteworthy that UFMC preserves the essential characteristics of OFDM, related to multiple-input multiple-output (MIMO) application.

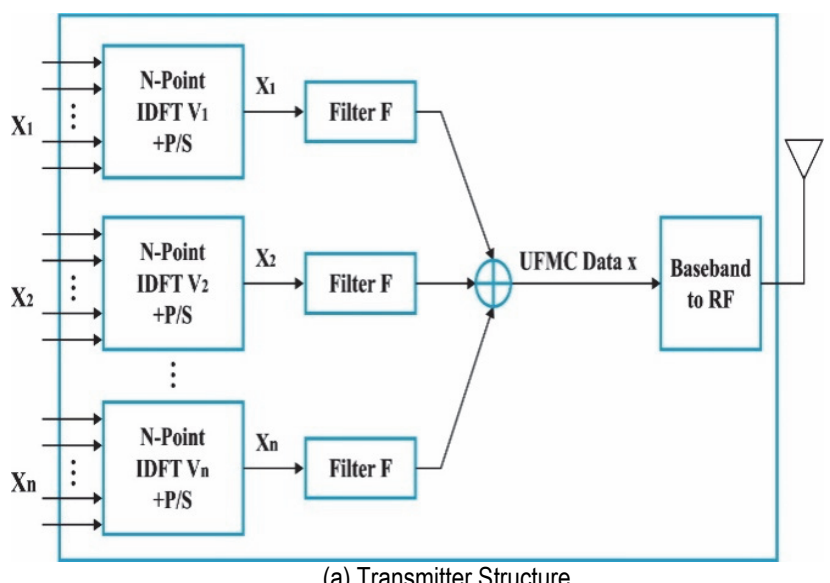

(a) Transmitter Structure

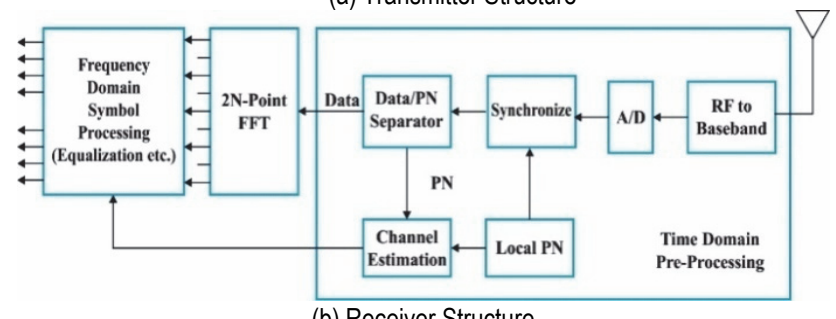

(b) Receiver Structure

Figure 1 General structure of UFMC system

Furthermore, in F-OFDM, the subbands are filtered later the addition of $\mathrm{CP}$ to find accurate frequency localization. Though this technique displays higher hardware difficulty, the filter tail extends to the following OFDM symbol. Nevertheless, UFMC has higher PAPR dependent on the utilization of multicarrier communication.

Various methods for PAPR reduction have been presented in the literature [4]. They can be categorized as encoding, signal distortion, and probabilistic methods. The initial technique utilizes filtering/clipping for limiting the 
maximal level of signal, henceforth decreasing the $P A P R$. It presents distortion in the communicated signal that demonstrates the bit error rate (BER) efficiency. Moreover, the encoded systems causes lower data rate effectiveness [20]. Additionally, the probabilistic approaches like partial transmit sequence (PTS) and selective mapping (SLM) present arbitrary stage changes on the communicated symbol as precoding step without affecting substantial distortion. It derives the cost of lower spectral efficiency (SE) due to side data transferred to the recipient for recovering the original symbol vector. Accordingly, several suggestions have been established in the study to improve the SE by attaining side data at the recipient site [3].

This paper develops an effective selective mapping with oppositional hosted cuckoo optimization (SMOHOCO) algorithm for $P A P R$ reduction in 5G UFMC models. During the SM-OHOCO algorithm, rather than the generation of several random phase sequences, SM$\mathrm{OHOCO}$ algorithm is performed repeatedly for attaining optimal solutions with minimal number of search iterations. As the optimization of phase sequence in the SLM technique is considered as an NP hard optimization problem, the OHOCO algorithm is applied, which integrates $\mathrm{HOCO}$ algorithm with oppositional based learning (OBL) strategy. For assessing the improved results of the presented SM-OHOCO algorithm, a series of simulations were carried out.

In short, the contribution of the paper is summarized as follows:

- Design a new SM-OHOCO) algorithm for $P A P R$ reduction in $5 \mathrm{G}$ UFMC systems.

- Perform iteratively to obtain optimal phase sequences with few searching iterations.

- Derive OHOCO algorithm by integrating the concepts of OBL with HOCO algorithm.

- Validate the performance of the SM-OHOCO algorithm in terms of diverse aspects.

The rest of the paper is arranged as follows. Section 2 briefs the existing PAPR reduction schemes for UFMC system. Section 3 introduces the SM-OHOCO model and experimental results are provided in section 4. Finally, section 5 concludes the paper.

\section{LITERATURE REVIEW}

Al-Rayif et al. [2] established a relative probabilistic $P A P R$ reduction method, known as decomposed SLM method for improving $P A P R$ in UFMC schemes. The idea relies on decomposing the complex symbol as to imaginary and real parts and later transforming every portion to various phase vectors to the Inverse Fast Fourier Transform (IFFT) process. In [12], a low computational complexity PTS is presented to reduce the $P A P R$ s of UFMC. Initially, it examines the variations in $P A P R$ among UFMC scheme and an orthogonal frequency division multiplexing method is used. Kumar et al. [8] presented a new PAPR reduction method that is most common requirement of 5G-based smart hospitals. The genetic algorithm (GA) with SLM called GA-SLM is recommended to reduce $P A P R$ of NOMA scheme. But the essential addition and multiplication for SLM methods are excessive that increases the system complexity.
Sarkar et al. [13] presented a Twin Symbol Hybrid Optimization utilized as a fundamental PTS (TSHO-PTS) technique of Cyclic Prefix-OFDM (CP-OFDM). It attains the needs of $5 \mathrm{G}$ telecommunication standard. Furthermore, the exhaustive searching for optimum phase factor can raise the computation cost of PTS. For overcoming this challenge, the hybrid version of Bald Eagle Search (BES) and slap swarm optimization (SSO) techniques are presented for investigating the optimum phase factor by PTS technique. In [14], an SLM is combined with UFMC to decrease the PAPR and $\mathrm{CM}$ without impacting the BER of $5 \mathrm{G}$ network. [7] proposed the signal scrambling method to decrease the PAPR in UFMC. The Shuffled Frog Leaping Algorithm (SFLA) is utilized as an optimizer for authenticating the whole search space for optimum phase vector with time limitations to generate the optimally altered UFMC signal with lesser PAPR.

Taşpınar and Şimşir [17] introduced a novel SLM system depending upon discrete forest optimization algorithm (DFOA) for UFMC system. The relevant system is made by embedding the DFOA to the traditional SLM with the aim of enhancing the value of phase factor with phase rotation model which is executed in frequency domain for reducing the $P A P R$ of final time-domain signal obtained from the SLM output. An effective PAPR reduction system named discrete cuckoo search based SLM (DCS-SLM) is recommended in [15]. When generating the presented DCS-SLM system, to create the actual CS technique to be combined with the traditional SLM technique, a new DCS method was established.

In [16], a novel discrete elephant herding optimization based PTS (DEHO-PTS) system is recommended to decrease the PAPR of UFMC signal to minimal level. Because of this certainty, the optimization phase of rotation factors is increased by the subblocks in the traditional method which is a combinatorial optimization problem to resolve distinct space. To combine the elephant herding optimization (EHO) technique to the PTS system, it established the distinct varieties of DEHO in this study. Taşpınar and Şimşir [17] presented a novel SLM method depending upon migrating birds optimization (MBO) method to provide an effective result to the problem of higher $P A P R$ in UFMC waveform. The MBO-based SLM (MBO-SLM) system for executing the efficient stage optimize from distinct space, cyclic bit flipping method is combined with the MBO technique.

\section{THE PROPOSED SM-OHOCO ALGORITHM}

Primarily, the transmission processes get initiated with the generation of QAM symbols in the data bits. Subsequent to the modulation procedure, the QAM symbols are separated to groups that are equivalent to the several subbands, and relevant symbol groups are deployed to several subbands.

$$
\begin{aligned}
& X_{b}(k)=\left\{\begin{array}{c}
\lambda(k), \text { if }(b-1) \leq k \leq b\left(\frac{N}{B}\right)-1 \\
0, \text { otherwise }
\end{array}\right. \\
& k=0,1,2, \ldots, N-1 ; b=0,1,2, \ldots, \mathrm{B}
\end{aligned}
$$


where the series of QAM symbol is accomplished in QAM modulator output and is denoted by $X(k)$, the frequency domain information is forwarded to subband $b$, denoted as $X_{b}(k), B$ denotes subband count, $k$ represents subcarrier index and $N$ indicates subcarrier count. Next to satisfying the deployment of symbol groups to $B$ subbands, IFFT is employed to all subband is given by:

$$
\begin{aligned}
& X_{b}(N)=\frac{1}{\sqrt{N}} \sum_{k=0}^{N-1} \lambda_{b}(k) e^{\frac{j 2 \pi k n}{N}} \\
& 0 \leq n \leq N-1 ; 1 \leq b \leq B
\end{aligned}
$$

where $X_{b}(n)$ denotes signal achieved by transforming $X_{b}(k)$ in the frequency to time domain by IFFT block in the subband $b$. Once the IFFT processes are executed at each subband, the related time-domain signal is exposed to the processes of cyclic prefix and filtering, correspondingly. Finally, the signal is attained from the output of finite impulse response filter that is included to obtain UFMC broadcast signal [18]. The adding and filtering processes on time-domain sub-band signals are equated by:

$$
s(n)=\sum_{b=1}^{B} X_{b}(n) \cdot f_{b}(n), 0 \leq n \leq N+L_{C P}+L_{f}-1
$$

where the impulse response belongs to the bth filter represented by $f_{b}(n) . L_{f}$ represents filter length and $L_{C P}$ indicates cyclic prefix length. Now, the $P A P R$ of broadcast signal is given by:

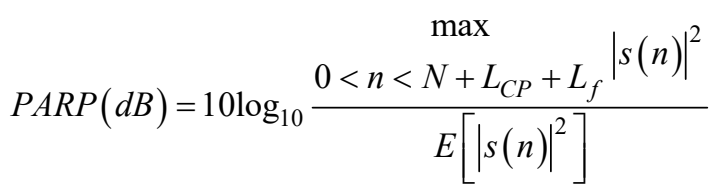

\subsection{Design of SLM Model for UFMC Systems}

The early process of SLM based PAPR design is for mapping the binary data to the series of QAM symbols determined as follows [18]:

$$
X(k)=[X(0), X(1), \ldots, X(N-1)]
$$

At the following phase, arbitrarily created $U$ distinct phase sequences $b^{(u)}(k)=\left[b^{(u)}(0), b^{(u)}(1), \ldots, b^{(u)}(N-1)\right]$, $u=0,1, \ldots, U \quad$ where $\quad b^{(u)}(k) \in\{-1,1\} \quad$ undergo multiplication with vector $X(k)$ in element wise method. Thereby, rotated data series can be attained by:

$$
\begin{aligned}
& X^{(u)}(k)=\left[X(0) \cdot b^{(u)}(0), X(1) \cdot b^{(u)}(1),\right. \\
& \left.\ldots, X(N-1) b^{(u)}(N-1)\right]= \\
& =\left[X^{(u)}(0), X^{(u)}(1), \ldots, X^{(u)}(N-1)\right]
\end{aligned}
$$

where $X^{(u)}(k)$ represents uth data series exposed to the phase rotation procedure by $u$-th phase sequence represented by $b^{(u)}(k)$. Consequently, $(L-1) N / 2$ zero are kept to right-handed and left-handed sides of $X^{(u)}(k)$ for oversampling procedure:

$$
\begin{aligned}
& X_{O S}^{(u)}(k)=\left[\frac{0,0, \ldots, 0}{(L-1) N / 2}, \frac{X^{(u)}(0), X^{(u)}(1), \ldots, X^{(u)}(N-1)}{N},\right. \\
& \left.\frac{0,0, \ldots, 0}{(L-1) N / 2}\right]
\end{aligned}
$$

where $L$ denotes oversampling rate. The resultant zero padded data series $X_{O S}^{(u)}(k)$ is later exposed to IFFT process for obtaining the time domain signal $X^{(u)}(n)$ given in Eq. (8):

$$
\begin{aligned}
& X^{(u)}(k)=\operatorname{IFFT}\left(X_{O S}^{(u)}(k)\right)=\frac{1}{\sqrt{N}} \sum_{k=0}^{N-1} X(k) . \\
& \cdot b^{(u)}(k) \cdot e^{\frac{j 2 \pi k n}{N}}, 0 \leq n \leq L N-1
\end{aligned}
$$

The optimum phase sequence guaranteeing the $X^{(u)}(n)$ to contain minimal PAPR value is determined using Eq (9):

$$
\begin{aligned}
& b^{*}(k)=\arg _{b^{(u)}(k)}^{\min }\left\{\begin{array}{c}
\max \\
0 \leq L N-1 \mid
\end{array} \mid \frac{1}{\sqrt{N}} \sum_{k=0}^{N-1} X(k) .\right. \\
& \left.\left.\cdot b^{(u)}(k) \cdot e^{\frac{j 2 \pi k n}{N}}\right|^{2}\right\}
\end{aligned}
$$

where $b^{*}(k)$ correspond to optimum $b^{(u)}(k)$ creating the expression value of $\arg \min \{\}$.$" operator minimal. In$ traditional SLM system, it necessitates transmitting the optimum phase sequence as side data which can obtain the input data at UFMC receiving end. The processes of the SLM block are ended by attaining optimum time-domain signal using $b^{*}(k)$ as given by:

$n^{*}(k)=\frac{1}{\sqrt{N}} \sum_{k=0}^{N-1} X(k) \cdot b^{*}(k) \cdot e^{\frac{j 2 \pi k n}{N}}, 0 \leq n \leq L N-1$

As the assignment of data to distinct UFMC sub-bands takes place from the frequency domain, the time-domain SLM-based optimum signals are afterward transformed to frequency domain using the FFT block. Next to the addition procedure of the filtered signals in many subbands, the resultant UFMC signal offers reduced PAPR.

\subsection{Algorithmic Design of OHOCO Algorithm}

HOCO algorithm is a recently presented optimization method [10] which is stimulated by the existence of a bird named cuckoo. These birds have the capacity for laying 
their eggs from the nest of other classes of birds. The cuckoo imitates the form and egg color of the host nest. Few restrictions have been made while utilizing this technique and enhanced for handling several challenges like system cost and availability, energy dispatch, job shop, cluster computing, and controller variables. In this study, the HOCO algorithm is developed to resolve the PAPR reduction in UFMC models. The important resolutions are created as nest and the eggs are laid in the nest of 3 major classes. The summary of this technique is given as follows.

Step 1. Input of the variables.

This step involves the input of maximal number of cuckoo generations $N_{\text {gen }}$ and the nest count $M$ is assumed as habitat.

Step 2. Produce the nest.

The nest is produced as follows.

$$
\left\{\begin{array}{l}
\operatorname{Nest}_{1}(r, n)=\left(r_{l}, r_{2}, \ldots, r_{m}, n_{l}, n_{2}, \ldots, n_{m}\right) \\
\operatorname{Nest}_{2}(r, n)=\left(r_{l}, r_{2}, \ldots, r_{m}, n_{l}, n_{2}, \ldots, n_{m}\right) \\
\operatorname{Nest}_{M}(r, n)=\left(r_{l}, r_{2}, \ldots, r_{m}, n_{l}, n_{2}, \ldots, n_{m}\right)
\end{array}\right.
$$

where $\operatorname{Nest}(n, r)$ represents a set of significant resolutions.

Step 3. Calculate the model consistency and restriction control [9].

$R_{s}(r, n)$

The restrictions are controlled by the succeeding penalty function:

$$
\begin{aligned}
& \bar{R}_{s}(r, n)=R_{s}(r, n)+\varnothing_{l} \cdot \operatorname{Max}\left\{0, g_{l}(r, n)-V\right\}+ \\
& +\varnothing_{2} \cdot \operatorname{Max}\left\{0, g_{2}(r, n)-C\right\}+\varnothing_{3} . \\
& \cdot \operatorname{Max}\left\{0, g_{3}(r, n)-W\right\}
\end{aligned}
$$

Step 4. Egg laying.

The eggs of cuckoos are laid based on the HOCO algorithm.

$$
\begin{aligned}
& E L R=\alpha \times \frac{\text { Number of current cuckoo'seggs }}{\text { Total number of eggs }} \times \\
& \times\left(V_{h i}-V_{\text {low }}\right)
\end{aligned}
$$

where $E L R$ represents laying radius, $\alpha$ indicates integer value, $V_{h i}$ and $V_{\text {low }}$ denotes upper and lower bound of the parameters, correspondingly. The number of eggs a cuckoo might lay are assumed from two to four.

Step 5. Chicks.

The study in [23] exposed that the cuckoo eggs are hosted by 3 major hosts and have distinct probabilities of success. Thus, in the current study, the cuckoo egg contains 3 distinct likelihoods for successful maturity, representing $\sigma_{1}, \sigma_{2}$ and $\sigma_{3}(0 \%, 100 \%)$, named quality of host. These values are arbitrarily created at every integer and generation. Thus, the nest is separated into 3 sets: $M_{1}, M_{2}$ and $M_{3}$, while the values are arbitrary. The quality of host is featured by:

$$
\left\{\begin{array}{c}
M_{l} \text { nests with } \sigma_{1} \text {, where } M_{1} \in\{M\} \\
M_{2} \text { nests with } \sigma_{2} \text {, where } M_{2} \in\left\{M-M_{1}\right\} \\
M_{3} \text { nests with } \sigma_{3} \text {, where } M_{3} \in\left\{M-M_{2}-M_{1}\right\}
\end{array}\right.
$$

Step 6. Migration.

The optimum generation of cuckoo migrates to other habitats that are the optimum resolutions and can be presented in the following generation to improve the resolution search.

Step 7. Repeating steps 2 to 6 till $N_{\text {gen }}$ is attained.

For improving the convergence rate of the $\mathrm{HOCO}$ model, the OBL concept is incorporated and OHOCO algorithm designed. Fig. 2 illustrates the flowchart of COA technique [11].

OBL denotes an optimization method that is utilized for several researches to enhance the quality of their initialized population solution by diversifying this solution. The OBL approach functions by searching both directions of the search space. These 2 directions are consisting of original and opposite solutions. Lastly, the OBL approach is taking the appropriate solution from every solution.

Opposite number: $x$ represents real number in the interval $x \in[l b, u n]$. The opposite number of $x$ is indicated by $\bar{x}$, defined in Eq. (15):

$\bar{x}=l b+u n-x$

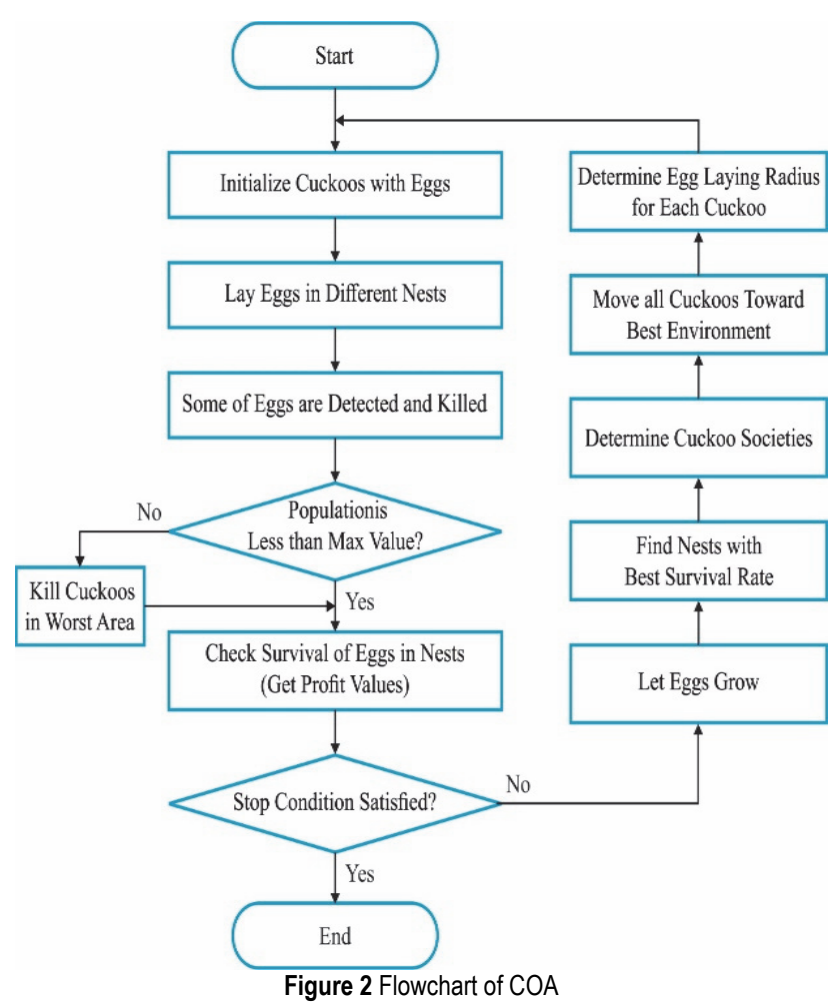

Eq (4) is generalized to employ search space with multi-dimension [19]. Thus, for generalizing, all search agents and opposite positions are given in succeeding Eq. (16) and Eq. (17):

$x=\left[x_{1}, x_{1}, x_{1}, \ldots, x_{D}\right]$ 
$\bar{x}=\left[\bar{x}_{1}, \bar{x}_{1}, \bar{x}_{1}, \ldots, \bar{x}_{D}\right]$

The values of every component in $\bar{x}$ is defined by Eq (18).

$\bar{x}_{j}=l b_{j}+u b_{j}-x_{j}$ where $j=1,2,3, \ldots, D$

Optimization Based on Opposite population: During this approach the FF represents $f($.$) . Hence, when the$ fitness value $f(\bar{x})$ of opposite solution is greater than $f(x)$ of its original solution $x$, next $x=\bar{x}$; or else $x=x$.

The process to integrate OBL with SSA is outlined in the subsequent steps:

1. Initiate the cuckoo location $X$ as $x_{i}$ where $(i=1,2, \ldots$, $n)$.

2. Define the opposite location of cuckoo population OX as $x_{i}$ where $(i=1,2, \ldots, n)$.

3. Choose the $n$ fittest nests from $\{X \cup O X\}$ and denote the novel population of HOCO algorithm.

\subsection{Design of SM-OHOCO Model for UFMC Systems}

The phase factors that need to be optimized in the PTS model takes either 0 or 1 . At the same time, the solution vectors of the OHOCO algorithm also comprise binary values. Therefore, it is feasible to apply the OHOCO algorithm to the $P A P R$ minimization problem and employ it in the phase optimization task. Besides, it is required to treat the 'zero' and 'one' from the solution space of the OHOCO model as $(-1,1)$ correspondingly. At the OHOCO technique based PTS technique, the position of the cuckoos is defined as the candidate phase sequences which undergo optimization using the following Eq. (19):

$b_{p}^{(v)}=\left[b_{p}^{(0)}, b_{p}^{(1)}, b_{p}^{(V-1)}\right], P=1,2, \ldots, P$

The processes involved in the phase sequence optimization using the SM-OHOCO algorithm in the PTS technique are listed as follows:

Step 1: Primarily, the initial population includes some phase sequences as provided in Eq. (19) produced in an arbitrary manner.

Step 2: Then, the set of arbitrary created phase sequences is validated by the use of the fitness function, as provided in Eq. (20):

$$
\begin{aligned}
& f i t\left(b_{p}^{(v)}\right)=\max _{0 \leq n \leq L N-1}\left|\sum_{v=0}^{V 1} b_{p}^{(v)} x^{(v)}(n)\right|, \\
& p=1,2, \ldots, P
\end{aligned}
$$

Step 3: Next to the computation of fitness values, the solutions get arranged based on the fitness values and divided into groups. The solution corresponding to the phase factor sequences in distinct nest is represented in Eq. (21):

$$
b_{c . e}{ }^{(v)}=\left|b_{c . e}{ }^{(0)}, b_{c . e}{ }^{(1)}, b_{c . e}{ }^{(V-1)}\right|, c=1,2, \ldots, C ; e=1,2
$$

Step 4: Every solution in the host nest performs the bit flipping task as given in Eq. (22):

$b_{c . e}^{\text {new }}=f l i p\left(b_{c e}\right)_{m}$

Here, the flipping function takes place by transforming the sign of the $m^{\text {th }}$ element in the sequence of $b_{c . e}$ as $b_{c . e} \in\{-1,1\}$. Next to the greedy selection process among the $b_{c . e}$ and $b_{c . e}^{\text {new }}$, the subsequent functions are employed to the variable $m$ for ensuring that the flipping procedure takes place in a cyclic way for all individuals as the iterations continue.

$m=m+1$

$m=\bmod (m-1, v)+1$

Step 5: Next to the termination of the solution update procedure, the migration phase has begun. The arbitrary set of solutions is created to replace the worst solutions in the nest, as defined in Eq. (25):

$$
\begin{aligned}
& b_{c . \text { worst }}^{(v)}=\operatorname{sgn}\left(\min \left(b_{c . e}^{(v)}\right)+\left(\max \left(b_{c . e}^{(v)}\right)-\right.\right. \\
& \left.-\min \left(b_{c . e}^{(v)}\right) \text { rand }\right)
\end{aligned}
$$

where $\min \left(b_{c . e}^{(v)}\right)$ and $\max \left(b_{c . e}^{(v)}\right)$ is equivalent to -1 and 1 , correspondingly. Here, sgn () function respective to signum function is employed for equalizing the arbitrarily created continuous values in the interval $\left[\min \left(b_{c . e}^{(v)}\right), \max \left(b_{c . e}^{(v)}\right)\right]$ to 1 or -1 as the arbitrary solution vectors are created instead of the worse solution consisting of 1 and -1 . In addition, the arbitrary numbers are denoted as $r$ and are created with the exception of 0.5 for preventing any dimensions of $b_{c \text {.worst }}^{(v)}$ equal to 0 .

Step 6: The migration process takes place and the optimal solution is saved.

Step 7: When the maximum iteration number (MIN) is attained, the procedure gets stopped. Else, go to step 3 .

\section{PERFORMANCE VALIDATION}

This section validates the performance of the proposed model under varying subcarriers such as 64 and 128 . Besides, the results are examined under $P A P R_{0}(\mathrm{~dB})-\mathrm{Pr}$ $(P A P R>P A P R 0)$. In addition, the results are also investigated under distinct modulation schemes.

Fig. 3 investigates the impact of subcarrier number on the $P A P R_{0}(\mathrm{~dB})-\operatorname{Pr}(P A P R>P A P R 0)$ curves of the proposed SM-OHOCO algorithm with the existence of 64 subcarriers. From the figure, it is obvious that the original model has failed to achieve an effective outcome over the other methods. At the same time, the MBO-SLM model 
has achieved considerably enhanced performance over the original one, but not the other methods. In addition, the BBA-SLM and GA-SLM models have exhibited considerably closer and moderate performance. Besides, the MBO-SLM model has tried to accomplish somewhat competitive performance over all the compared methods except the SM-OHOCO technique. However, the SMOHOCO technique outperformed the other methods under varying $P A P R_{0}(\mathrm{~dB})$.

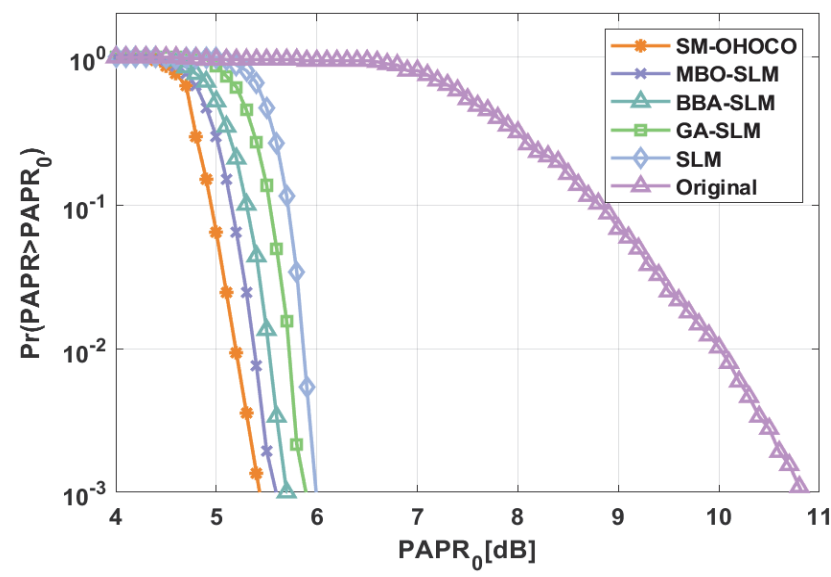

Figure 3 Result Analysis of $P A P R_{0}(\mathrm{~dB})$ with $\operatorname{Pr}\left(P A P R>P A P R_{0}\right)$ on 64 subcarriers

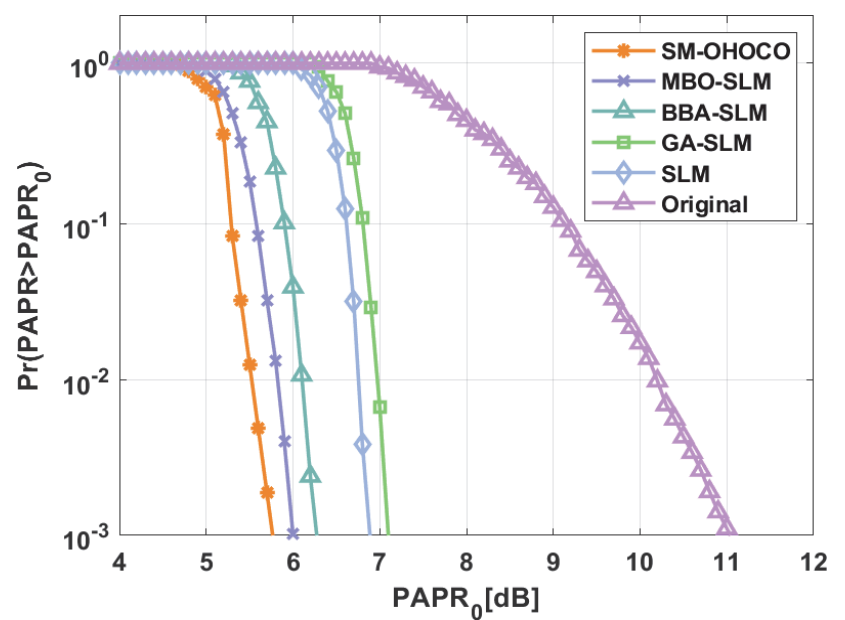

Figure 4 Result Analysis of PAPRO $(\mathrm{dB})$ with $\operatorname{Pr}(P A P R>P A P R 0)$ on 128 subcarriers

Fig. 4 determines the impact of subcarrier number on the PAPR0 (dB)-Pr $(P A P R>P A P R 0)$ curves of the proposed SM-OHOCO algorithm with the existence of 128 subcarriers. From the figure, it can be revealed that the original model has failed to achieve effective results over the other methods. Concurrently, the MBO-SLM technique has achieved considerably enhanced performance over the original one, but not the other methods. In addition, the BBA-SLM and GA-SLM models have showcased considerably closer and moderate performance. Besides, the MBO-SLM model has tried to accomplish somewhat competitive performance over all the compared methods except the SM-OHOCO method. But, the SM-OHOCO technique outperformed the other techniques under varying $P A P R_{0}(\mathrm{~dB})$.

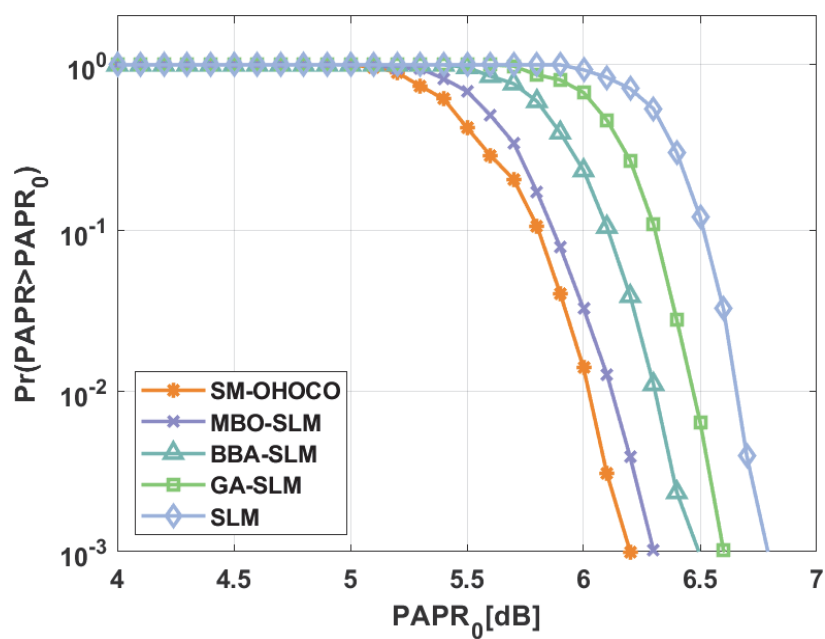

Figure 5 Result analysis of $P A P R O(\mathrm{~dB})$ with $\operatorname{Pr}(P A P R>P A P R O)$ on 4-QAM modulation schemes

Fig. 5 examines the impact of modulation schemes on the $P A P R_{0}(\mathrm{~dB})-\operatorname{Pr}(P A P R>P A P R 0)$ curves of the proposed SM-OHOCO algorithm with the existence of 4QAM modulation schemes. From the figure, it is clear that the original model has failed to achieve an effective outcome over the other methods. Simultaneously, the MBO-SLM model has achieved considerably enhanced performance over the original one, but not the other approaches. In addition, the BBA-SLM and GA-SLM models have exhibited considerably closer and moderate performance. Besides, the MBO-SLM model has tried to accomplish somewhat competitive performance over all the compared methods except the SM-OHOCO technique. However, the SM-OHOCO technique outperformed the other methods under varying $P A P R_{0}(\mathrm{~dB})$.

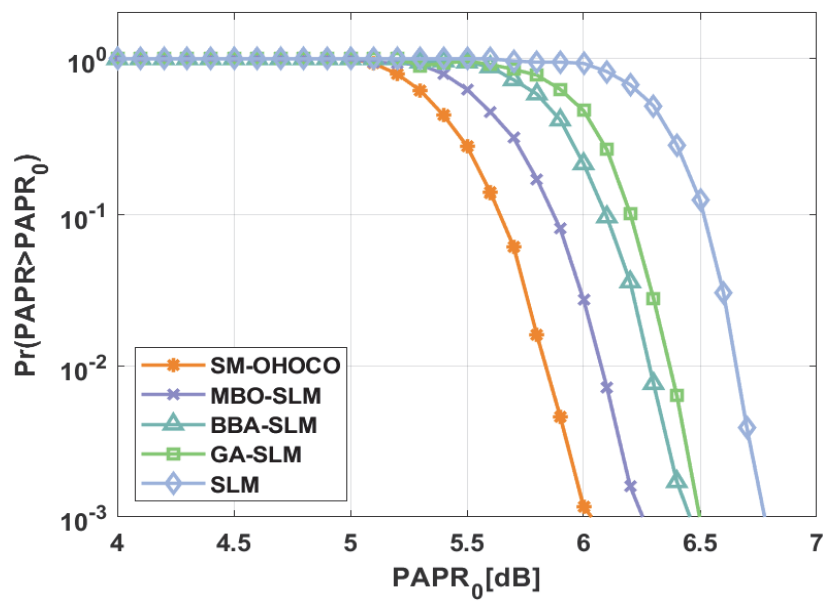

Figure 6 Result Analysis of PAPRO $(\mathrm{dB})$ with $\operatorname{Pr}(P A P R>P A P R 0)$ on 16 - QAM modulation schemes

Fig. 6 explores the impact of modulation schemes on the $P A P R_{0}(\mathrm{~dB})-\operatorname{Pr}(P A P R>P A P R 0)$ curves of the proposed SM-OHOCO model with the existence of 16QAM modulation schemes. From the figure, it is stated that the original model has failed to achieve an effective outcome over the other methods. At the same time, the MBO-SLM model has achieved considerably enhanced performance over the original one, but not the other methods. Also, the BBA-SLM and GA-SLM approaches have exhibited considerably closer and moderate performance. Moreover, the MBO-SLM model has tried to 
accomplish somewhat competitive performance over all the compared methods except the SM-OHOCO technique. However, the SM-OHOCO model demonstrated the other techniques under varying $P A P R_{0}(\mathrm{~dB})$.

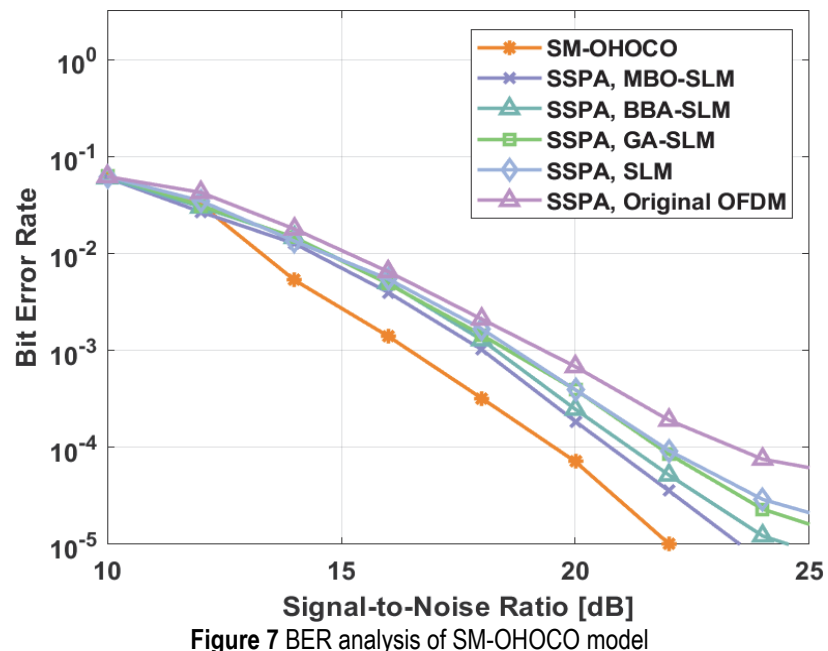

Fig. 7 investigates the BER analysis of the presented SM-OHOCO technique with other existing techniques under varying SNR. From the figure, it is identified that the SM-OHOCO technique has resulted in an effective outcome by offering the lowest SNR. At the same time, the SSPA, SSPA-SLM, and SSPA-GA-SLM models have exhibited poor performance by accomplishing slightly closer and higher BER. At the same time, the SSPA, SSPABBA-SLM, and SSPA-MBO-SLM models have demonstrated competitive outcomes with the moderate BER. However, the proposed SM-OHOCO technique has exhibited superior results by attaining a lower BER. For instance, with the SNR of $15 \mathrm{~dB}$, the SM-OHOCO technique has obtained a minimal BER of $10^{-2.5}$ whereas the other methods such as SSPA-MBO-SLM, SSPA-BBASLM, SSPA-GA-SLM, SSPA-SLM, and SSPA-OFDM models have resulted in a maximum BER of $10^{-2.2}, 10^{-2.15}$, $10^{-2.1}, 10^{-2.1}$, and $10^{-1.95}$ respectively. Followed by, with the SNR of $20 \mathrm{~dB}$, the SM-OHOCO technique has reached a minimal BER of $10^{-4.2}$ whereas the other models such as SSPA-MBO-SLM，SSPA-BBA-SLM， SSPA-GA-SLM, SSPA-SLM, and SSPA-OFDM models have resulted in a maximum BER of $10^{-3.7}, 10^{-3.6}, 10^{-3.41}, 10^{-3.4}$, and $10^{-3.1}$ correspondingly. At last, with the SNR of $25 \mathrm{~dB}$, the SMOHOCO model has attained a lesser BER of $10^{-6}$ whereas the other approaches like SPA-MBO-SLM, SSPA-BBASLM, SSPA-GA-SLM, SSPA-SLM, and SSPA-OFDM methodologies have resulted in a maximal BER of $10^{-5}, 10^{-}$ $5,10^{-4.7}, 10^{-4.6}$, and $10^{-4.2}$ correspondingly. From the above mentioned experimental analysis, it is apparent that the proposed SM-OHOCO model has obtained effective $P A P R$ reduction in the $5 \mathrm{G}$ UFMC systems. The enhanced performance is due to the iterative attainment of optimal phase sequences, inclusion of OBL concept in $\mathrm{HOCO}$ algorithm.

\section{CONCLUSION}

This paper has developed a novel SM-OHOCO method for $P A P R$ reduction in $5 \mathrm{G}$ UFMC models. The SMOHOCO algorithm gets executed repeatedly for attaining optimal solutions with minimal number of search iterations. The early process of SLM based PAPR design is for mapping the binary data to the series of QAM symbols. As the optimization of phase sequence in the SLM technique is considered as an NP hard optimization problem, the OHOCO method is applied. For improving the convergence rate of the $\mathrm{HOCO}$ technique, the OHOCO algorithm is derived by incorporating the concept of OBL with $\mathrm{HOCO}$ algorithm. For assessing the improved results of the presented SM-OHOCO algorithm, a series of simulations were carried out. The proposed model can be implemented in real time applications to accomplish high data rate communications. The experimental outcomes stated that the presented model has demonstrated promising results compared to other existing methods in terms of distinct measures. In future, new hybridization of two metaheuristic optimization algorithms can be designed to improve the performance of the HOCO algorithm.

\section{REFERENCES}

[1] Al-Rayif, M. I., Seleem, H. E., Ragheb, A. M., \& Alshebeili, S. A. (2020). PAPR Reduction in UFMC for 5G Cellular Systems. Electronics, 9(9), 1404. https://doi.org/10.3390/electronics9091404

[2] Almutairi, A. F., Al-Gharabally, M., \& Krishna, A. (2019). Performance analysis of hybrid peak to average power ratio reduction techniques in 5G UFMC systems. IEEE Access, 7, 80651-80660. https://doi.org/10.1109/ACCESS.2019.2916937

[3] Elhelw, A. M. \& Badran, E. F. (2015). Semi-Blind Error Resilient SLM for PAPR Reduction in OFDM Using Spread Spectrum Codes. PLoS ONE., 10(5), 127639. https://doi.org/10.1371/journal.pone.0127639

[4] Fathy, S. A., Ibrahim, M., El-Agooz, S., \& El-Hennawy, H. (2020). Low-Complexity SLM PAPR Reduction Approach for UFMC Systems. IEEE Access., 8, 6802-68029. https://doi.org/10.1109/ACCESS.2020.2982646

[5] Hasan, N. U., Ejaz, W., Ejaz, N., Kim, H. S., Anpalagan, A., \& Jo, M. (2016). Network selection and channel allocation for spectrum sharing in $5 \mathrm{~g}$ heterogeneous networks. IEEE Access, 4(6), 980-992. https://doi.org/10.1109/ACCESS.2016.2533394

[6] Kasmi, M., Mhatli, S., Bahloul, F., Dayoub, I., \& Oh, K. (2020). Performance analysis of UFMC waveform in graded index fiber for $5 \mathrm{G}$ communications and beyond. Optics Communications, 454, 124360. https://doi.org/10.1016/j.optcom.2019.124360

[7] Krishna Kishore, K. \& Rajesh Kumar, P. (2020) Comprehensive Analysis of PAPR Reduction in OFDM, UFMC and Signal Scrambling UFMC Technique using Shuffled Frog Leaping Algorithm. IETE Journal of Research, 12(2), 1-12. https://doi.org/10.1080/03772063.2020.1816225

[8] Kumar, A., Bhargav, A., Karthikeyan, A., Rajagopal, K., Srinivasan, A. K., \& Tsegay, A. N. (2021) Low Computational Artificial Intelligence Genetic Algorithm Assisted SLM PAPR Reduction Technique for Upcoming 5G Based Smart Hospital. In Metaheuristic and Evolutionary Computation: Algorithms and Applications, Springer. 555-567. https://doi.org/10.1007/978-981-15-7571-6_25

[9] Mellal, M. A., Al-Dahidi, S., \& Williams, E. J. (2020) System reliability optimization with heterogeneous components using hosted cuckoo optimization algorithm. Reliability Engineering \& System Safety., 203, 107110. https://doi.org/10.1016/..ress.2020.107110

[10] Mellal, M. A. \& Williams, E. J. (2015). Cuckoo optimization algorithm with penalty function for combined heat and 
power economic dispatch problem. Energy, 93(15), 17111718. https://doi.org/10.1016/j.energy.2015.10.006

[11] Rajabioun, R. (2011). Cuckoo optimization algorithm. Applied soft computing, 11(8), 5508-5518. https://doi.org/10.1016/j.asoc.2011.05.008

[12] Rong, W., Cai, J., \& Yu, X. (2017). Low-complexity PTS PAPR reduction scheme for UFMC systems. Cluster Computing, 20(4), 3427-3440. https://doi.org/10.1007/s10586-017-1124-9

[13] Sarkar, M., Kumar, A., \& Maji, B. (2021). PAPR reduction using twin symbol hybrid optimization-based PTS and multichaotic-DFT sequence-based encryption in CP-OFDM system. Photonic Network Communications, 41(2), 148-162. https://doi.org/10.1007/s11107-020-00923-7

[14] Shawqi, F. S., Audah, L., Mostafa, S. A., Gunasekaran, S. S., Baz, A., Hammoodi, A. T., Alhakami, H., Hassan, M. H., Jubair, M. A., \& Alhakami, W. (2020). A new SLM-UFMC model for universal filtered multi-carrier to reduce cubic metric and peak to average power ratio in $5 \mathrm{G}$ technology. Symmetry, 12(6), 909. https://doi.org/10.3390/sym12060909

[15] Şimşir, Ş. \& Taşpınar, N. (2020). A novel discrete cuckoo search algorithm-based selective mapping technique to minimize the peak-to-average power ratio of universal filtered multicarrier signal. International Journal of Communication Systems, 33(18), 4640. https://doi.org/10.1002/dac.4640

[16] Şimşir, Ş. \& Taşpınar, N. (2021). A novel discrete elephant herding optimization-based PTS scheme to reduce the PAPR of universal filtered multicarrier signal. Engineering Science and Technology, an International Journal. https://doi.org/10.1016/j.jestch.2021.03.001

[17] Taşpınar, N. \& Şimşir, Ş. (2021). Advanced SLM scheme based on discrete forest optimization algorithm for PAPR minimization in UFMC waveform. Wireless Networks, 27(2), 1353-1368. https://doi.org/10.1007/s11276-020-02515-9

[18] Taşpınar, N. \& Şimşir, Ş. (2020). An efficient SLM technique based on migrating birds optimization algorithm with cyclic bit flipping mechanism for PAPR reduction in UFMC waveform. Physical Communication, 1(43), 101225. https://doi.org/10.1016/j.phycom.2020.101225

[19] Tubishat, M., Idris, N., Shuib, L., Abushariah, M. A., \& Mirjalili, S. (2020). Improved Salp Swarm Algorithm based on opposition based learning and novel local search algorithm for feature selection. Expert Systems with Applications, 145(12), 113122.

https://doi.org/10.1016/j.eswa.2019.113122

\section{Contact information:}

A. JAYAMATHI, Assistant Professor

(Corresponding author)

Department of ECE,

K.S.R College of Engineering,

Tiruchengode, India

E-mail: jayamathi2013@gmail.com

T. JAYASANKAR, PhD, Assistant Professor (Sr.Gr)

Department of ECE,

University College of Engineering,

BIT Campus,

Anna University,

Tiruchirappalli, India

E-mail: jayasankar27681@gmail.com

K. VINOTH KUMART, PhD, Associate Professor

Department of Electronics \& Communication Engineering,

SSM Institute of Engineering and Technology,

Dindigul, Tamil Nadu, India-624002

E-mail: vinodkumaran87@gmail.com 